\title{
Acoustic Effects Accurately Predict an Extreme Case of Biological Morphology
}

\author{
Zhiwei Zhang, ${ }^{1}$ Son Nguyen Truong, ${ }^{2}$ and Rolf Müller ${ }^{1,3, *}$ \\ ${ }^{1}$ School of Physics, Shandong University, Hongjia Lou 5, 250100 Jinan, China \\ ${ }^{2}$ Institute of Ecology \& Biological Resources, Vietnamese Academy of Science \& Technology, Hanoi, Vietnam \\ ${ }^{3}$ Department of Mechanical Engineering, Virginia Tech \& Institute for Advanced Learning and Research, \\ 150 Slayton Avenue, Danville, Virginia 24540, USA. \\ (Received 4 January 2009; revised manuscript received 9 May 2009; published 14 July 2009)
}

\begin{abstract}
The biosonar system of bats utilizes physical baffle shapes around the sites of ultrasound emission for diffraction-based beam forming. Among these shapes, some extreme cases have evolved that include a long noseleaf protrusion (sella) in a species of horseshoe bat. We have evaluated the acoustic cost function associated with sella length with a computational physics approach and found that the extreme length can be predicted accurately from a fiducial point on this function. This suggests that some extreme cases of biological morphology can be explained from their physical function alone.
\end{abstract}

DOI: 10.1103/PhysRevLett.103.038701

PACS numbers: $43.80 .+\mathrm{p}$

Extremes in biological form have intrigued researchers at least since Darwin observed the exceptionally long spur of an orchid flower [1]. This interest is justified by the potential role that such outliers can play as model systems to identify particularly strong and unmitigated driving forces of evolution [2]. However, biological evolution is a complicated process that is subject to limitations imposed by inheritance, selection, development, and design [3]. As a consequence, it is still a matter of debate whether morphological features should be seen as optimal adaptations and could be predicted from an analysis of their function alone $[4,5]$.

The present work investigates the possibility that a physical effect by itself could predict extreme cases in biological morphology accurately and hence offer a sufficient parsimonious explanation for them. The model system studied here for this purpose is the shape of a biological structure with a well-defined physical function: The nostrils of bat species that emit their ultrasonic biosonar pulses through them are surrounded by so-called "noseleaves". These structures serve a physical (acoustic) function as beam forming baffles for the biosonar ultrasound beam and are among the most striking features in the exterior morphology of these animals. A noseleaf feature common to all the approximately 80 species of old-world horseshoe bats (genus Rhinolophus, [6,7]) is a central pegshaped forward protrusion of the noseleaf called a "sella" (Fig. 1). The horseshoe bat species studied here, Bourret's horseshoe bat (Rhinolophus paradoxolophus, stands out among its congeneric peers as an extreme case of noseleaf morphology: The sella has reached an exceptional length and the posterior noseleaf part behind it (the lancet) is greatly reduced (Fig. 1). The specific name "paradoxolopus" refers to this noseleaf shape, underscoring its novelty among horseshoe bats [8]. Several comparative studies have already pointed to the general importance of the dimensions of noseleaf protrusions in bats: In the newworld leaf-nosed bats (Phyllostomidae), a comparative analysis has suggested that long lancet-shaped processes are among the evolved noseleaf features [9] and most likely to correlate with ecological factors $[10,11]$. These findings propound the hypothesis that the length of the noseleaf sella in Bourret's horseshoe bat (Fig. 1) could play a key role in the sensory ecology of this species, but do not offer a functional explanation.

In the present work, a computational physics approach was chosen to evaluate the acoustic properties of the noseleaf as a function of sella length within a range from almost no sella to well beyond the sella lengths known from any extant horseshoe bat species [6]. The objective of this approach was to test the hypothesis that these acoustic properties could define a cost function that predicts the exceptionally long natural sella length by virtue of some fiducial point. In order to combine controllable quantitative experimentation [12] with the full complexity of the natural shapes, high-resolution, three-dimensional digital models of noseleaf shapes from three specimens of Bourret's

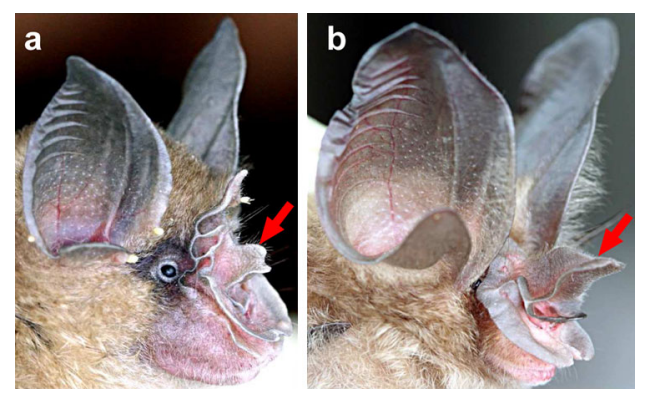

FIG. 1 (color online). Bourret's horseshoe bat (Rhinolophus paradoxolophus) has an extraordinarily long noseleaf sella: (a) a typical horseshoe-bat noseleaf (sella length $2.7 \mathrm{~mm}$ ) in the Bigeared horseshoe bat (Rhinolophus macrotis), (b) Bourret's horseshoe bat (sella length $9.1 \mathrm{~mm}$ ). In each image, the sella is marked by an arrow. The two species shown are closely related and both belong to the taxonomic "philippinensis group". Measured by maximum forearm length, the Big-eared horseshoe bat is only about $16 \%$ smaller than Bourret's horseshoe bat [6]. 
horseshoe bat were obtained using microcomputer tomography [13]. A geometrical scaling operation was then applied to the sellas of these noseleaf models: Voxels representing sella tissue were hand-marked in the tomographic model of the noseleaf. The scaling direction $\left(\overrightarrow{\mathbf{v}}_{\mathrm{scl}}\right)$ was found as the principal direction of the point cloud (P) containing the positions $\left(x_{i}, y_{i}, z_{i}, i=1 \ldots N\right)$ of the $N$ sella voxels in Cartesian space from the relationships:

$$
\begin{gathered}
\mathbf{P}=\left[\begin{array}{ccc}
x_{1}-\bar{x} & \ldots & x_{N}-\bar{x} \\
y_{1}-\bar{y} & \ldots & y_{N}-\bar{y} \\
z_{1}-\bar{z} & \ldots & z_{N}-\bar{z}
\end{array}\right], \quad \mathbf{C}=\frac{1}{N} \mathbf{P P}^{T}, \\
\mathbf{C} \overrightarrow{\mathbf{v}}_{\mathrm{scl}}=\lambda_{\max } \overrightarrow{\mathbf{v}}_{\mathrm{scl}},
\end{gathered}
$$

where $\bar{x}, \bar{y}, \bar{z}$ are the mean values of the three voxel coordinates (e.g., $\bar{x}=\frac{1}{N} \sum_{1}^{N} x_{n}$ ), $\mathbf{C}$ is the $3 \times 3$ covariance matrix of the point coordinates and $\lambda_{\max }$ its largest eigenvalue.

The positions of all voxels marked as sella tissue were subjected to linear scaling along this principal direction $\left(\overrightarrow{\mathbf{v}}_{\mathrm{scl}}\right)$. To preserve the solid nature of the sella tissue even under elongation, neighborhood relationships between sella voxels (in an eight-neighborhood) were recorded prior to scaling. After elongation, this information was used to reconnect all former neighbors by ray-tracing the connections between them. That is, for two former neighbor voxels with grid indices $\left(i_{l}, j_{l}\right)$ and $\left(i_{u}, j_{u}\right)$ and positions $\overrightarrow{\mathbf{p}}_{\left(i_{i}, j_{l}\right)}, \overrightarrow{\mathbf{p}}\left(i_{u}, j_{u}\right)$ after scaling, a voxel with position $\overrightarrow{\mathbf{p}}(i, j)$ was marked as tissue if its distance to the connecting line between the voxels was below a threshold value $\left(d_{\max }\right)$, taken to be one voxel edge length: i.e.,

$$
\frac{\left|\left[\overrightarrow{\mathbf{p}}\left(i_{u}, j_{u}\right)-\overrightarrow{\mathbf{p}}\left(i_{l}, j_{l}\right)\right] \times\left[\overrightarrow{\mathbf{p}}\left(i_{l}, j_{l}\right)-\overrightarrow{\mathbf{p}}(i, j)\right]\right|}{\left|\overrightarrow{\mathbf{p}}\left(i_{u}, j_{u}\right)-\overrightarrow{\mathbf{p}}\left(i_{l}, j_{l}\right)\right|} \leq d_{\max } \wedge i_{l} \leq i \leq i_{u} \wedge j_{l} \leq j \leq j_{u} .
$$

The distance threshold $\left(d_{\max }\right)$ was experimentally determined to fill in all holes in the elongated shapes.

Sixteen sella lengths equally spaced on a linear length axis from one-eighth to twice the original length of the sella in Bourret's horseshoe bat were created for each of the three noseleaves (Fig. 2). This neighborhood covers the entire region of the domain of the acoustic cost function in which the evolution of sella length in the horseshoe bat has taken place (as documented by the known extant species [6]). All scaled digital shape representations were used to formulate a two-step numerical model $[13,14]$ that predicts the acoustic beam forming behavior of the original and modified noseleaves in the acoustic far field. The acoustic near field due to two point sources placed in the nostrils was predicted for the nodes of finite-element mesh by a numerical solution to the Helmholtz equation [13]. Cubic linear finite elements bounded by a layer of threedimensional mapped wave-envelope infinite elements were used for this purpose [13]. From the surface of the finite-element domain, the wave was projected outward to a sphere in the far field using a Kirchhoff integral formu-

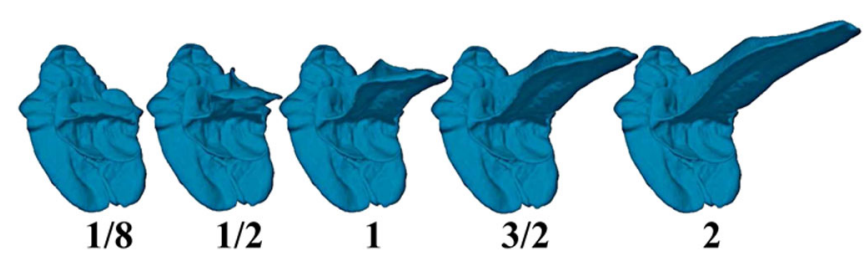

FIG. 2 (color online). Scaling of the sella allows controlled experimentation while retaining natural shape complexity: The examples of scaled digital noseleaf representations shown are derived from a single specimen of Bourret's horseshoe bat. The scaling factor of each derived shape relative to the original sella is indicated below the shape. The natural length of the sella shown [the same as in Fig. 1(b)] was $9.1 \mathrm{~mm}$; the sellas of the other two noseleaves studied were 7.8 and $8.2 \mathrm{~mm}$ long. lation [15]. The field amplitudes found on the sphere surface were taken as the directivity gains [13]. Predictions of the beam patterns were made for discrete frequencies that covered the bands used by the biosonar pulses of the specimens and other individuals from the same population in steps of $1 \mathrm{kHz}$. These pulses were found to consist of three harmonics with "constantfrequency frequency-modulated (CF-FM)" timefrequency contours [16] and frequencies of maximum energy (corresponding to the constant frequency portions of the pulse) at 27,54 , and $81 \mathrm{kHz}$. The analyzed frequency bands were 20 to $30 \mathrm{kHz}, 50$ to $55 \mathrm{kHz}$, and 77 to $82 \mathrm{kHz}$, respectively. This fundamental frequency is low compared to other Rhinolophid species [8].

The effect of sella length on the sonar beam differed pronouncedly between these three harmonics: For the lowest harmonic $(27 \mathrm{kHz})$, the beam pattern contained a single, comparatively broad lobe that underwent only minor changes in flank shape in response to scaling of the sella. The overall angular extent of these lobes remained largely unchanged (Fig. 3). In contrast to this, strong effects of sella length on the ultrasonic beam patterns were found for the second and third harmonics. For the second harmonic $(54 \mathrm{kHz})$, the vertical (elevation) width of the beam decreased monotonically with increasing sella length. This behavior is consistent with basic physical principles: The larger an aperture is relative to the wavelength, the narrower a beam it is capable of producing [17]. It is also consistent with this expectation that the narrowing of the beam is confined to the vertical dimension, since the sella enlarges the aperture around the nostrils only dorsally but not laterally. The vertical beamwidth achieved at a sella scale of one $\left(18.9^{\circ}\right.$ at $90 \%$ of the maximum gain) is comparatively narrow. For example, in the rufous horseshoe bat (Rhinolophus rouxi), a beamwidth of $34^{\circ}$ has been found at the slightly higher frequency of $60 \mathrm{kHz}$ [13]; a 


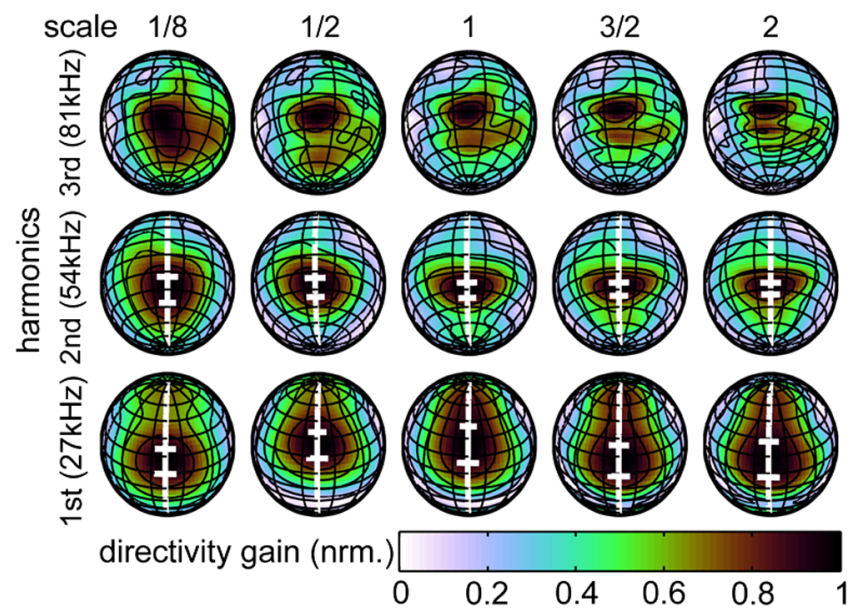

FIG. 3 (color online). Sella scale affected the beam pattern of the three harmonics in different ways as evident by directivity examples obtained at different sella scales (columns) for the three harmonics of the biosonar pulse (rows): Beam gain (individually normalized for each beam pattern) is coded linearly by color ( $s$ scale bar). Gain contours are spaced in increments of 0.2 and grid lines in increments of $15^{\circ}$ for latitude and longitude. The white meridian marks the azimuth of the maximum beam gain for the first and second harmonic. The white horizontal bars indicate the $90 \%$-of-maximum beam gain points between at which beamwidth was measured.

similar beamwidth has been measured in the greater horseshoe bat (Rhinolophus ferrumequinum) at an even higher frequency [18].

The most conspicuous feature of the predicted beam patterns is that the slope of the decrease in vertical beamwidth underwent a rapid quantitative change around a sella scale of one [i.e., the natural sella length, s. Fig. 4(a)]. Above as well as below the natural sella length, the relationship between sella scale and average beamwidth (averaged over individual and frequency within the harmonic) was found to be approximately linear (correlation coefficients $r=0.927$ and $r=0.982$ above and below a scale of one, respectively). Furthermore, if the average beamwidth data is approximated by a two-segment piecewise linear function, placing the border between the two line segments at a noseleaf scale of one minimizes the residual of the data fit [Fig. 4(b)]. This was exactly true for all but two of the 30 equally-spaced directivity gain thresholds at which beamwidths were measured between 0.7 (i.e., approximately $-3 \mathrm{~dB}$ ) and 0.99 , making this behavior a uniform property of the entire beam tip above (and also slightly below) the $-3 \mathrm{~dB}$ level. For the two exceptional amplitude thresholds, the minimum-residual segment border position were also not far from the original sella length (at scales of 1.125 and 1.25). Best placement of the segment border at or near the original sella size was also found in each individual specimen. For two of the three specimens, the best placement was exactly at the original sella for almost all threshold (24 and 26 out of the 30 amplitude thresholds, respectively). For the third specimen, the scale of the seg- 2nd harmonic
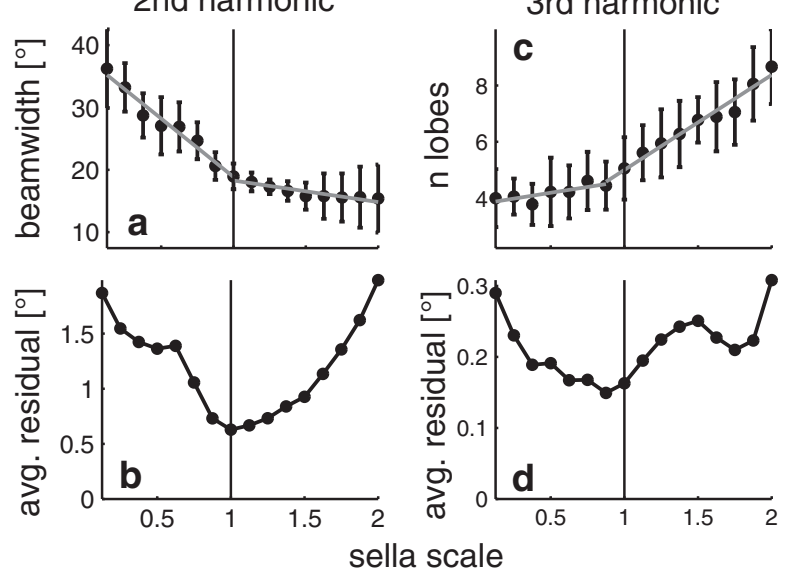

FIG. 4. The natural length of the sella is a fiducial point in the relationship between sella scale and beamwidth: (a) average beamwidth at an amplitude threshold of $90 \%$ of maximum beam gain, with averaging over specimen and frequency (2nd harmonic: 6 frequencies from 50 to $55 \mathrm{kHz}$ ); error bars depict the standard deviation, (b) average residual of a two-segment piecewise linear fit to beamwidth as a function of sella scale, the residual is minimized if the border between the segments is placed at the natural sella length, (c) average number of lobes along the beam pattern cross section in the 3rd harmonic (6 frequencies from 77 to $82 \mathrm{kHz}$ ) defined by the meridian through the global beam gain maximum; error bars depict the standard deviation, (d) average residual of a two-segment piecewise linear fit to average lobe number as a function of sella scale.

ment border that minimized the residual was most frequently placed at the scale of 1.25 (20 out of 30 thresholds) and 6 times at a scale of one. Below a scale of one, the slope of the linear fit for beamwidth as a function of sella scale is $-18.7^{\circ}$. Hence, scaling the sella from $1 / 8$ th of the natural length up to the full natural length changes the average 90\%-of-maximum beamwidth from $36.2^{\circ}$ to $18.9^{\circ}$. For scales above one, the slope is only $-3.4^{\circ}$ and hence doubling the length of the sella only reduces the width of the beam by that number. Therefore, as the sella length increases beyond what is seen in nature, it enters into a different regime, where further length increases only have a small, most likely negligible, impact on focusing the biosonar beam of the second harmonic.

It may be speculated that a larger sella length is associated with some cost not immediately related to sensing, such as energy expenditure or loss of maneuverability. In this scenario, the large gains in focusing achievable below the actual length could be hypothesized to offset these costs, whereas the small gains achievable above it would not. But in addition to these hypothetic possibilities, there is also an acoustic effect that may encumber the sensory function of noseleaves with longer sellas: In the beam pattern of the third harmonic, a longer sella length manifests itself in the breakup of the single mainlobe (which is flanked by smaller sidelobes) into several narrower lobes (Fig. 3). The number of these lobes increases with increas- 
ing sella length [Fig. 4(c)]. A breakup of the beam pattern into isolated lobes could be a disadvantage for uses that require the type of narrowly focused beam that is produced by the second harmonic. If counted by the number of local maxima present in the beam pattern along the elevation dimension, lobe breakup starts already at sella scales slightly below the natural value [Fig. 4(d)]; for the average number of lobes the best border point for a two-segment linear fit was found at a sella scale of 0.875 . Visual inspection of all the individual cases averaged here supports the notion that this breakup indeed happens at slightly lower sella lengths than the transition in the slope of the beamwidth of the second harmonic most of the time. However, the extent to which the utility of a focused beam pattern is affected by a breakup into lobes is not as unequivocally quantified as the focusing capabilities are by the beamwidth. Besides the number of local maxima, their closeness to each other in amplitude and the depth of the notches separating them may also play a role. Therefore, it is difficult to say, whether the small distance between the two fiducial points could be attributed any significance.

Our data show, however, that the natural sella length falls into a fairly narrow region of scales in which the relationship between sella scale and beam pattern undergoes a qualitative and quantitative transition. This is evident for all studied specimens and frequencies individually as it is from the overall averages. The unified focused beams that can be found for the second and third harmonics only in this region could give the animals several sensory advantages: Within the extent of the beam, the directivity gain increases the signal-to-noise ratio and hence the biosonar's operating range and signal characterization capabilities. The use of comparatively low frequencies by this species could also indicate a comparatively large biosonar operating range since lower frequencies suffer considerably less from atmospheric sound absorption $\left(\propto f^{2}\right)$. The downside of a focused beam is that a wider angular coverage can only be achieved through scanning. Scanning is time consuming, but could be used as a source for information on target direction $[19,20]$ and to separate targets from clutter in narrow passageways. The importance of a narrow beam at comparatively low frequencies is also consistent with the rudimentary nature of the noseleaf lancet and particularly its furrows in Bourret's horseshoe bat. The furrows of the lancet have been shown to act as resonance cavities that widen the biosonar beam at low frequencies of the biosonar pulse in other horseshoe bat species [21]. Such a function would contradict the effect of the long sella in Bourret's horseshoe bat and may be precluded anyway by strong shadowing from the large sella. It may thus be hypothesized that the sensory ecology of Bourret's horseshoe bat differs from that of other horseshoe bats in some crucial way that demands for a lowfrequency yet focused biosonar beam.

Under the assumption that these advantages add to the animals' sensory capabilities and thereby their fitness, it may be hypothesized that the transition region evident in beamwidth and lobe number has acted as a stationary point for the evolution of the noseleaf of Bourret's horseshoe bat. For sella lengths that fall below or above this region, directional selection would have driven the population's noseleaf scale into this region and stabilizing selection would have kept it there. The sella length of Bourret's horseshoe bat could thus be a model case in which quantitative data supports the notion that basic physical constraints on sensing alone can be sufficient to predict the outcome of evolution. However, the existence of ecological advantages to a narrower beam in Bourret's horseshoe bat still needs experimental verification.

Supported by the European Union (CILIA project), the Natural Science Foundation of China (Project No. 10774092), and Shandong University (Taishan Fund).

*rolf.mueller@vt.edu

[1] C. Darwin, The Various Contrivances by which Orchids are Fertilized by Insects (John Murray, London, 1862).

[2] J. B. Whittall and S. A. Hodges, Nature (London) 447, 706 (2007).

[3] S. J. Arnold, Am. Nat. 140, S85 (1992).

[4] G. Parker and J.M. Smith, Nature (London) 348, 27 (1990).

[5] R. M. Alexander, Nature (London) 412, 591 (2001).

[6] C. Csorba, P. Ujhelyi, and N. Thomas, Horseshoe Bats of the World (Alana Books, Bishop's Castle, Shropshire, UK, 2003).

[7] Mammal Species of the World. A Taxonomic and Geographic Reference, edited by D. E. Wilson and D. M. Reeder (Johns Hopkins University Press, Baltimore, MD, 2005), 3rd ed..

[8] J. L. Eger and M. B. Fenton, Mammalian Species 1 (2003).

[9] A.L. Wetterer, M. V. Rockman, and N.B. Simmons, Bulletin of the American Museum of Natural History 248, 1 (2000).

[10] H. T. Arita, J. Mammal. 71, 36 (1990).

[11] W. Bogdanowicz, R. D. Csada, and M. B. Fenton, J. Mammal. 78, 942 (1997).

[12] W. J. Bock, American Zoologist 20, 217 (1980).

[13] Q. Zhuang and R. Müller, Phys. Rev. E 76, 051902 (2007).

[14] R. Müller, J. Acoust. Soc. Am. 116, 3701 (2004).

[15] O. M. Ramahi, IEEE Trans. Antennas Propag. 45, 753 (1997).

[16] G. Jones and E. C. Teeling, Trends Ecol. Evol. 21, 149 (2006).

[17] J. W. Goodman, Introduction to Fourier Optics (Roberts and Company Publishers, Greenwood Village, Colorado, 2005), 3rd ed..

[18] H.-U. Schnitzler and A. D. Grinnell, J. Comp. Physiol. A 116, 51 (1977).

[19] J. Mogdans, J. Ostwald, and H.-U. Schnitzler, J. Acoust. Soc. Am. 84, 1676 (1988).

[20] V. A. Walker, H. Peremans, and J. C. T. Hallam, J. Acoust. Soc. Am. 104, 569 (1998).

[21] Q. Zhuang and R. Müller, Phys. Rev. Lett. 97, 218701 (2006). 